\title{
EL JUEGO, ESPACIO NATURAL PARA FAVORECER EL DESARROLLO MOTOR
}

\author{
Martha Tobón de Awad \\ Terapista ocupacional, Universidad Nacional \\ Por un Mañana
}

\section{Sinopsis}

El juego es la principal ocupación del niño. Por esto aprovecha cualquier situación para jugar. Al hacerlo aprende a manejar el mundo que le rodea y adquiere habilidades para interactuar con el ambiente. En la etapa escolar el énfasis del juego infantil está en las actividades de movimiento. Jugando, el niño ejercita sus patrones motores y les imprime coordinación y destreza. Además recibe una gran variedad de estímulos sensoriales provenientes del ambiente. Estos estímulos favorecen el desarrollo del esquema corporal, la organización del espacio y la base postural. Por estos motivos puede ser útil ofrecer a maestros alternativas de enseñanza cuyo eje sea la actividad lúdica. y aprovechar el espacio de la escuela para proponer programas de estimulación de las habilidades motoras en términos de juego.

Palabras claves: Estimulación por el juego. Desarrollo sensoriomotor. Comportamiento ocupacional del niño. Juego y desarrollo motor.

El propósito de este artículo es argumentar la utilidad del juego como estrategia para favorecer el desarrollo motor del niño en la escuela. Siendo el juego su principal ocupación, puede ser útil ofrecer alternativas de enseñanza centradas en la actividad lúdica, ya que es en la etapa escolar cuando se perfeccionan ciertas habilidades de movimiento. Con esta propuesta, las clases cuyas actividades tienen que ver con el aprendizaje motor se pueden convertir en las favoritas de niños y niñas. 
La experiencia de la autora; durante 11 años, en el área de educación le ha permitido acopiar evidencia sobre la utilidad del juego para favorecer el desarrollo motor. Además esta estrategia hace parte del Programa de estimulación sensoriomotora del Jardín Infantil Por Un Mañana, el cual desarrolla su actividad pedagógica con el modelo de Pedagogía Participativa (Amaya, Lizarazo, otros, 1996)

El fundamento teórico de los argumentos que se presentan en este artículo, sigue los lineamientos del modelo de Ocupación Humana.(Kielhofner, 1992. Fisher, 1991), de la teoría de Integración Sensorial (Ayres, 1979. Fisher, 1991) y de la teoría de juego (Bundy, 1995).

Primero, se resalta la relación del juego con el comportamiento ocupacional del niño: Luego, se describen algunos eventos del desarrollo motor en relación con la actividad lúdica. Posteriormente, se muestra la importancia del ambiente de juego como fuente de estimulación sensorial. Finalmente, se ejemplifica la utilización del juego como estrategia de estimulación en el espacio de la escuela.

\section{Comportamiento ocupacional y juego}

"IBueno niños dejen la jugadera que hoy no han hecho nada!" Esta frase se oye con frecuencia calificando el tiempo que los niños dedican al juego. Para algunos adultos el juego infantil es una pérdida de tiempo. Es tan poco importante que puede ser interrumpido para hacer otras cosas. Sin embargo, para el niño, el juego es una ocupación seria, importante y necesaria. Autores como Fisher (1991), Bundy (1995) y Reilly (1974), han estudiado el juego y su significado para el niño. Por ejemplo Reilly describió el juego como un proceso de maestría para lograr el desarrollo motor, la resolución de problemas y la socialización: durante el juego los niños repiten acciones logrando mayor 
habilidad corporal; juegan para imitar a los adultos en las actividades de la vida diaria buscando controlar su ambiente y repiten los sucesos que han vivido para entenderlos.

Diversos autores han afirmado que los niños dedican gran parte del día al juego, pues es su principal ocupación (Kielhofner, 1992. Fisher, 1991 y Bundy, 1994). Por ejemplo Bundy resalta cómo para el niño el juego es una actividad placentera. Por eso, su disposición para jugar parte de su propia motivación y el tiempo que dedica al juego depende de sí mismo y no de lo que sucede a su alrededor. El juego es la base del comportamiento ocupacional del niño. Este se define como el conjunto de actividadés que desarrollan las personas durante su vida y que le dan significado a su existencia. Estas actividades son el trabajo, el autocuidado y las actividades de tiempo libre (Kielhofner, 1992). El comportamiento ocupacional del niño consiste en las acciones que desarrolla para adaptarse al mundo que le rodea. Estas acciones son juego, autocuidado y aprendizaje (Fisher, 1991).

Por otra parte, al jugar los niños buscan comprender el mundo que los rodea y responder en forma adecuada a las exigencias del ambiente (McEwen, 1990). Además, cuando los niños juegan, desarrollan habilidades sociales, cognitivas y del lenguaje (Reilly, 1974). Por ejemplo, cuando el niño juega al señor que va manejando a la oficina, ensaya los roles de los adultos, habla como los papás y dramatiza las situaciones que ha vivido.

Los juegos estimulan el desarrollo de las habilidades sensoriomotoras puesto que el interés del niño al jugar se constituye en el motor que lo lleva a ejercitar diversos patrones motores como saltar, correr o trepar (Bundy, 1991). Además la interacción del niño con los objetos del ambiente favorece la adecuada madurez de los sistemas sensoriales -táctil, vestibular, propioceptivo, visual y auditivo-(Fisher 1991). 
Esta información le permite al niño, por ejemplo, desarrollar el esquema corporal, gracias a las sensaciones que recibe a través de su piel, sus movimientos y su visión.

En la etapa escolar los niños están en el proceso de desarrollar sus habilidades motoras. Por este motivo, permanecen en constante movimiento y buscan actividades que aumenten sus destrezas. Cuando los niños están motivados por el juego, lo repiten. muchas veces y desarrollan así mayor habilidad corporal. Por ejemplo a la mayoría de los niños les encanta el recreo: corren, saltan, se cuelgan y ejercitan muchas veces una secuencia motora. Además, crean nuevos esquemas de movimiento para jugar golosa, saltar lazo o practicar fútbol. Para ellos, el recreo es un espacio de actividades libres que parten del propio interés, enmarcada en la magia del juego.

Partiendo de las anteriores consideraciones, es de gran utilidad aprovechar el interés que tienen los niños por el juego, como recurso de enseñanza. Muchas actividades escolares pueden ser planteadas en términos de juego. Por ejemplo, en la clase de educación física se pueden trabajar esquemas motores de coordinación auditiva - motora a través de rondas y juegos tradicionales como «arroz con leche" o «materile».

La utilización del juego como recurso pedagógico puede ayudar al maestro a cumplir con los objetivos de su clase, ya que le permite plantear el trabajo de la clase de una forma divertida. Además, facilita la motivación del niño para superar los retos que propone el maestro y favorece la repetición de las actividades, elemento esencial para consolidar lo aprendido.

Los niños aprovechan cualquier lugar, objeto y compañero para jugar. Esto se denomina ambiente de juego (Reily, 1974). En 1983 Webstu describió el ambiente como las condiciones, circunstancias e influencias que rodean y 
afectan el desarrollo de un organismo. En el juego, el ambiente está conformado por las Condiciones del lugar, o sea los espacios y los objetos; por las personas o compañeros de juego y por las situaciones o juegos (Ritcher y Oetter, 1990). En la escuela, dichos elementos del ambiente de juego se identifican con facilidad:

* El gimnasio, el patio de recreo y el aula son los espacios. Allí se despierta el interés del niño por explorar invitándolo a jugar.

* Los amigos y los maestros son las personas. Estas acompañan al niño durante las actividades y se convierten en parte importante del escenario de juego.

* Los materiales didácticos, equipos deportivos y elementos del patio son los objetos. Estos invitan al niño a interactuar para manipularlos.

El maestro puede preparar el ambiente de su clase para facilitar el aprendizaje, proponer ideas, preparar actividades y favorecer la magia del juego. Muchos elementos del salón o del patio se pueden emplear por ejemplo, para saltar, atravesar o evadir. La disposición que se le dé al salón promoverá el interés del niño por explorar. La decoración y los objetos son importantes pues pueden favorecer la organización y disposición para interactuar. También se puede organizar la situación para que el.juego dé el resultado pedagógico que se busca. Por ejemplo proponer el juego de golosa como actividad para aprender a saltar en un pie. En la gráfica No. 1 se ilustra la influencia del ambiente de juego en el desarrollo del comportamiento ocupacional del niño. 


\section{AMBIENTE DE JUEGO}

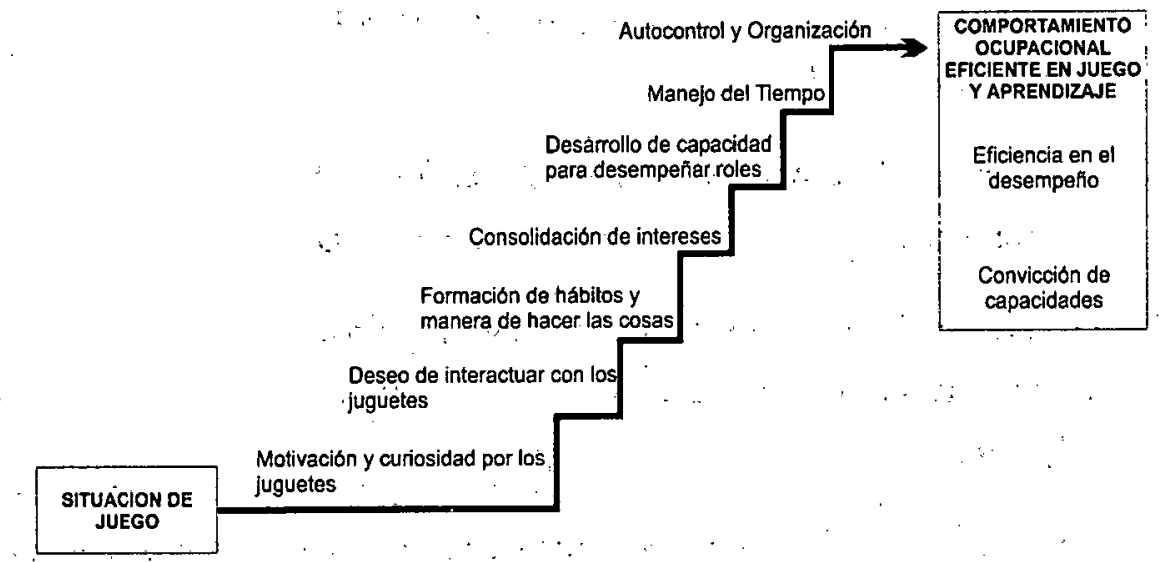

Gráfica No. 1 Influencia del ambiente de juego en el desarrollo del comportamiento ocupacional del niño.

Una situación de juego promueveve la curiosidad e interés por los juguetes llevando al niño a interactuar con el ambiente. En la medida en que juega; séleccioná preferencias y manieras particulares de hacer las cosas. Además afianza habilidades motoras, comunicativas y de pensamiento. Jügando asume roles y aprende a organizarse. Cada vez que su intervención es exitosa, el niño percibe sus capacidadés y desarrolla un comportamiento ocupacional eficiente para jugar $y$ aprender:

En síntesis, el juego es la principal ocupación del niño; para él es una actividad placentera que le permite aprender y desarrollar habilidades de movimiento. Para el maestro el juego es un buen recurso de enseñanza, ya que le permite plantear a sus alumnos las actividades en términos interesantes, que promuevan la participación y el aprendizaje. 


\section{Desarrollo motor y juego}

Desde que nace el niño, inicia el control de los movimientos de su cuerpo gracias al interés que tiene por interactuar con el ambiente que lo rodea (Fisher, 1991). Por el deseo de jugar, adquiere nuevos patrones motores y mejora su postura y equilibrio. Durante los juegos perfecciona sus habilidades. motoras, las combina y les imprime coordinación y destreza.

El desarrollo motor se inicia con movimientos generales como por ejemplo, extender el cuerpo contra la gravedad. Se perfecciona con el manejo armónico de los dos lados del cuerpo. Esto facilita, por ejemplo, el uso de las manos y permite definir la preferencia manual. Y se completa con patrones específicos tales como saltar, pararsè en un pié, manipular objetos o utilizar las manos para escribir o recortar. (Gassier, 1985)

El desarrollo motor es un proceso espiral y progresivo de adquisición de patrones como arrastrarse, sentarse, gatear, caminar, correr, saltar y trepar. Con cada nuevo patrón, el niño puede interactuar con su ambiente con mayor facilidad y acceder a juegos mas elaborados (Bundy, 1995). Por ejemplo, cuando el niño aprende a gatear puede alcanzar los juguetes que están fuera de su alcance para explorarlos.

De igual forma, la prensión tiene una secuencia precisa: alcanzar, agarrar, soltar, lanzar y hacer pinza para manipular objetos pequeños (Gassier, 1993), la cual se estimula por las exigencias del ambiente. En la gráfica no. 2 se muestra la influencia del ambiente de juego en el proceso de desarrollo motor. 


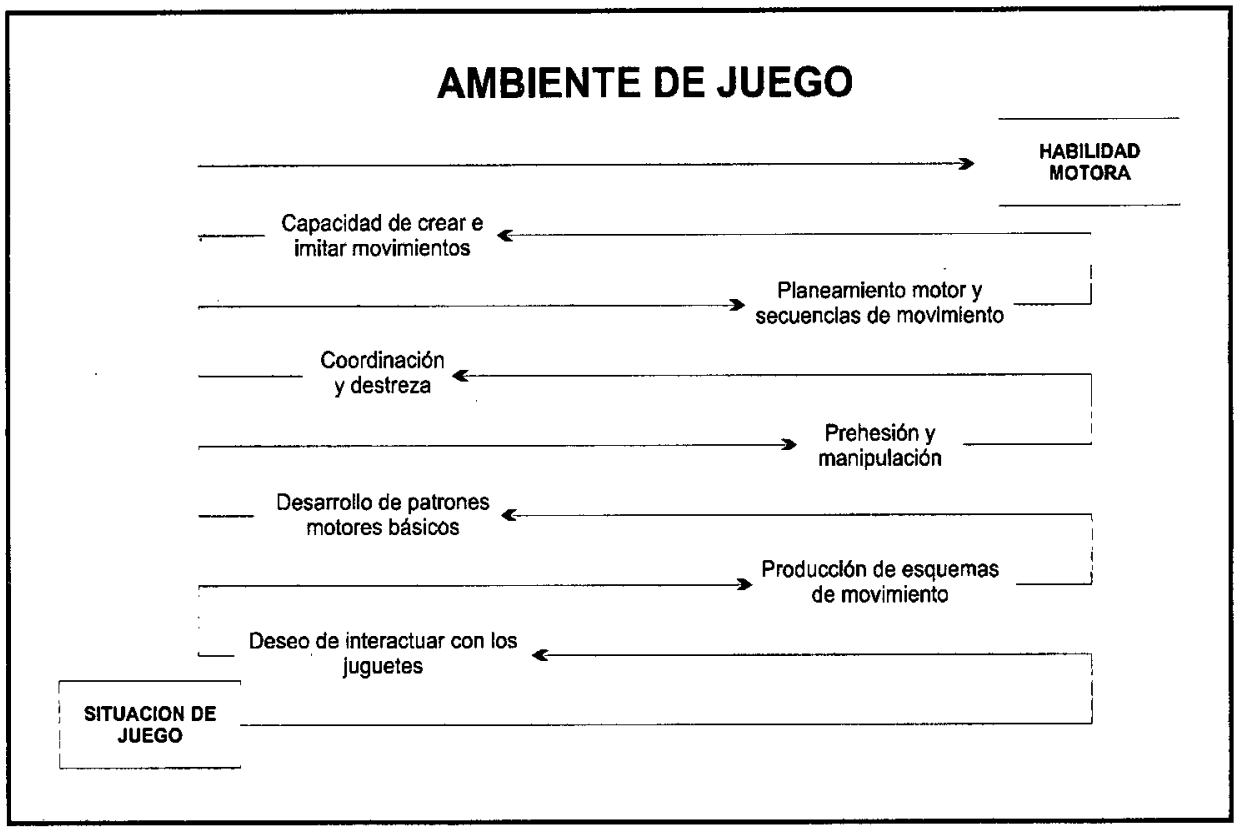

Gráfica No. 2. Influencia del ambiente de juego en el desarrollo motor

Cualquier propuesta de juego motiva al niño a interactuar con el ambiente. Favorece la aparición de patrones motores básicos. Exige la creación de secuencias de movimiento. Promueve el planeamiento de nuevos esquemas motores y facilita la manipulación de los objetos. Con base en estas adquisiciones el niño puede imitar movimientos y mejorar su coordinación y destreza. El proceso se completa cuando el niño es hábil para planear sus movimientos e interactuar con el ambiente.

En la etapa escolar el niño perfecciona sus habilidades motoras básicas gracias a las diversas actividades que realiza: Inicia destrezas de autocuidado, como vestido, alimentación e higiene y se hace más independiente. Planea nuevos esquemas de movimiento y desarrolla su coordinación y destreza al jugar durante el recreo. Perfecciona su habilidad manual, pues aprende a manejar 
élementos escolares como arcilla, plastilina, crayolas o colores y las tareas escolares propician la repetición de movimientos. Las clases de educación Física, expresión corporal o arte favorecen el afianzamiento de las habilidades motoras de los niños. Los maestros pueden proponer actividades dirigidas a mejorar la coordinación y destreza motora en términos de juego.

Los niños generalmente están dispuestos a participar en los juegos y esto facilita al maestro el cumplimiento de sus objetivos de tipo motor. Por ejemplo, en la clase de educación física, se pueden proponer juegos de buscar pistas siguiendo caminos de obstáculos donde los niños deben pasar en diferentes posiciones (parados, gateando, arrastrándose, etc.). También es valioso abrir espacios de juego libre dentro de las clases para observar a qué juegan los niños y qué actividades lúdicas prefieren. Posteriormente el maestro puede programar en su clase esos juegos para aprovechar los intereses de los niños.

Como resumen se puede decir que el niño logra desarrollar sus habilidades motoras al enfrentarse a los diferentes retos que le plantea la escuela. Cuando los retos se presentan en el contexto del juego, el niño se motiva a enfrentarlos con mayor facilidad. Por lo tanto; los maestros pueden utilizar el recurso del juego para favorecer el desarrollo motor. Los juegos se pueden escoger de acuerdo con las exigencias que proponen, la opción de estimulación que ofrecen y el grado de control que exigen del niño.

\section{Estimulación sensorial y juego}

Para adquirir patrones motores eficientes, el niño necesita de la integración y organización de varios estímulos sensoriales. Este proceso de recepción, relación e interpretación de estímulos se denomina integración sensorial (Ayresw 1979). La organización e interpretación de la información produce como resultado comportamientos adaptativos. Estos son la repuesta adecuada 
del niño a las exigencias del ambiente. En el cuadro No.1, aparecen los diferentes tipos de estímulos sensoriales, sus receptores y su función.

\begin{tabular}{|c|c|c|}
\hline SISTEMA SENSORIAL & RECEPTORES & FUNCIÓN \\
\hline TACTIL & $\begin{array}{l}\text { En la piel: Corpúsculos de Meissner } \\
\text { Terminaciones nerviosas libres }\end{array}$ & $\begin{array}{l}\text { Tacto discriminativo. Esterognosis. } \\
\text { Temperatura. Dolor. Esquema corporal. } \\
\text { Expresión afectiva } \\
\text { (Caricia) }\end{array}$ \\
\hline $\begin{array}{l}\text { PROPIOCEPTIVO } \\
\cdot ; \\
\cdot\end{array}$ & $\begin{array}{l}\text { En músculos, teridones y } \\
\text { articulaciones: } \\
\text { Organo tendinoso de Golgi. Husos } \\
\text { musculares. Terminaciones nerviosas } \\
\text { intra-articularés }\end{array}$ & $\begin{array}{l}\text { Contracción muscular. Estiramiento de } \\
\text { tendones. Posición de articulaciones. } \\
\text { Sensación de movimiento, posición y peso. } \\
\text { Esquema corporal. }\end{array}$ \\
\hline VESTIBULAR & $\begin{array}{l}\text { En el oído interno: Canales } \\
\text { semicirculares. Sáculo y Utriculo. }\end{array}$ & $\begin{array}{l}\text { Sensación de movimientos, posición de la } \\
\text { cabeza en el espacio. Aceleración y } \\
\text { desaceleración del movimiento. } \\
\text { Sensación de enderezamiento contra la } \\
\text { fuerza de gravedad. Postura. Equilibrio. } \\
\text { Movimientos de los ojos. Relaciones } \\
\text { espaciales. }\end{array}$ \\
\hline VISUAL & Oio & $\begin{array}{l}\text { Percepción de forma y color. Relaciones } \\
\text { espaciales. Percepción visual. }\end{array}$ \\
\hline AUDITIVO & Oido & $\begin{array}{l}\text { Percepción del sonido. Tono. Timbre. } \\
\text { lntensidad. Percepción auditiva. }\end{array}$ \\
\hline
\end{tabular}

Cuadro No. 1 Principales sistemas sensoriales con sus receptores y función.

Ante cualquier situación, el niño recibe información sensorial de origen táctil, vestibular, propioceptiva, visual y auditiva. Dicha información es relacionada e interpretada en su cerebro produciendo una respuesta adaptativa acorde con la situación (Ayres, 1979. Fisher, 1991). Por ejemplo, cuando el niño juega fútbol, mira el balón (información visual). Lo toca con el pié (información táctil). Se mueve para empujarlo (información vestibular y propioceptiva): Escucha al amigo que se lo pide (información auditiva). Y lanza el balón haciendo un buen pase (comportamiento adaptativo). 
Un adecuado proceso de integración sensorial permite entre otros logros, desarrollar el conocimiento del mapa corporal, alcanzar una adecuada base postural y lograr la organización espacial:

* El mapa corporal es el producto de las sensaciones que se reciben a través de la piel. Se desarrolla, por ejemplo, cuando el niño juega con crema, arena o pintura. Esta imagen mental del cuerpo es básica para planear movimientos e interactuar con el ambiente.

* La base postural, es la suma de reacciones de equilibrio, enderezamiento y estabilidad muscular. Se desarrolla por ejemplo, cuando el niño juega en columpios, balancines o barra de equilibrio. Gracias a la base postural; el niño puede moverse con agilidad y mantener sú posición ante las exigencias de la fuerza de gravedad.

* La organización espacial es la capacidad de orientarse y relacionarse con los objetos del medio ambiente. Se estimula, por ejemplo, con juegos de pistas o con el ordenamiento de objetos. El manejo del espacio permite al niño desarrollar su direccionalidad y ubicación. En la Gráfica No. 3 se ilustra la importancia del ambiente de juego como fuente de estimulación sensorial.

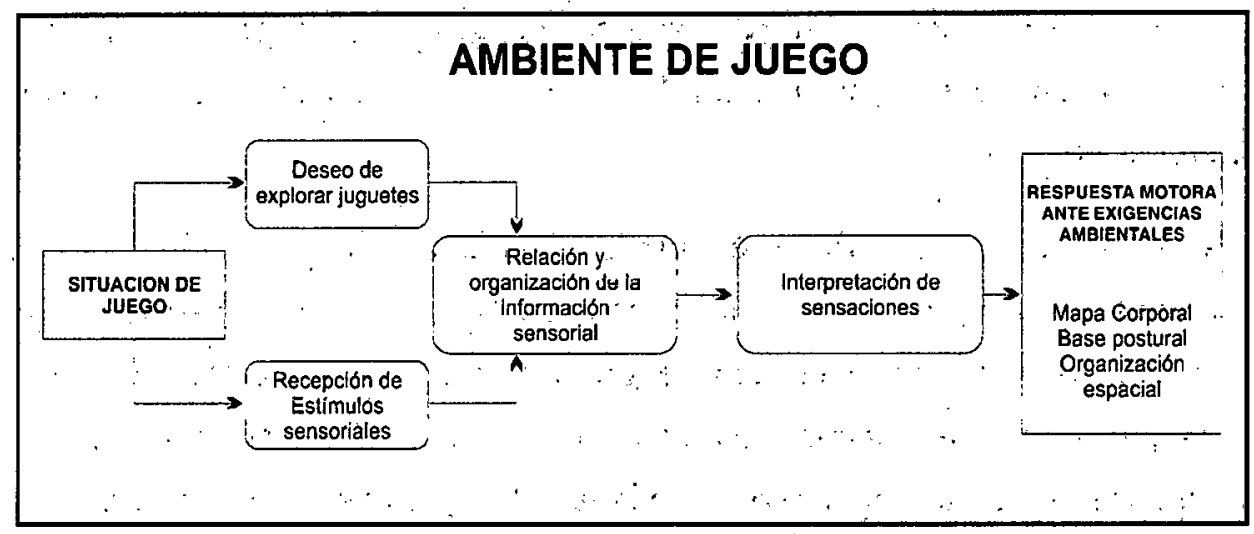

Gráfica No. 3 Importancia del ambiente de juego como fuente de estimulación serisorial. 
El deseo de explorar el ambiente invita al niño a interactuar con éste. Esta interacción le brinda estímulos sensoriales que son organizados e interpretados en el cerebro. Las habilidades corporales se desarrollan como resultado del proceso de integración sensorial. El niño responde con actos motores que son comportamientos adaptativos a las exigencias del ambiente.

En la escuela las opciones de interacción con el ambiente se amplían. El niño se relaciona y juega con otros niños, aprende nuevos juegos y recibe una rica estimulación sensorial. Los maestros pueden favorecer la estimulación sensorial con la preparación dé ambientes y juegos que ofrezcan los estímulos táctiles, vestibulares, propioceptivos, visuales y auditivos.

Por ejemplo. En la clase de artes, el niño maneja variados materiales tales como arcilla, témpera, plastilina, aserrín, etc. El maestro puede aprovechar para que los niños analicen los materiales e identifiquen a través del tacto, su textura, temperatura y consistencia. Esta actividad ayudará al niño a mejorar el tacto y la percepción de las manos. Además al manipular los materiales mejorará su destreza manual.

Los maestros también pueden preparar el ambiente de la clase con diversos materiales para que el niño perciba diferentes sensaciones al jugar. Por ejemplo, pisos de diversas texturas (tapete, baldosa, colchonetas de espuma) que permitan sensaciones táctiles. $O$ columpios y balancines que permitan percibir el movimiento.

Para recapitular, el niño recibe en forma permanente la información sensorial proveniente de los objetos que le rodean. Esta información se organiza en el cerebro, lo cual le permite comprender la situación y responder en forma adaptativa ante las exigencias ambientales. Gracias a la integración de los estímulos sensoriales el niño desarrolla por ejemplo el esquema corporal, la 
base postural y la organización en el espacio. Los maestros pueden favorecer la madurez sensorial del niño a través de los juegos que propongan en sus clases.

\section{Propuesta de estimulación motora en el contexto del juego}

Para desarrollar un programa de estimulación motora (Amaya, Lizarazo y otras, 1996) con base en actividades lúdicas los maestros pueden proponer los siguientes objetivos, como complemento de los propios de su cláse:

* Permitir al niño disfrutar de la magia del juego

* Facilitar el conocimiento del ambiente que lo rodea

* Favorecer la madurez sensorial

* Promover patrones motores propios de la edad

* Desarrollar el planeamiento motor, el movimiento creativo y la expresión corporal

* Promover la coordinación y la destreza motora.

El programa se desarrolla a partir de la observación que se hace de los niños en situaciones de juego. Esta observación permite al maestro conocer el nivel de desarrollo sensoriomotor de su grupo. Se puede realizar durante el recreo y en las clases que tienen que ver con actividades motoras como educación física, danza, expresión corporal o artes y en salón de clases.

Los prótagonistas del programa de estimulación son: los niños, los maestros, los terapeutas que trabajan en la escuela y los padres de familia. Los niños son los actores principales. Los maestros facilitan las propuestas de juego y preparan el ambiente. Los terapeutas diseñan las actividades de acuerdo 
con el nivel de desarrollo. Y los padres de familia multiplican dichas actividades a través de planes caseros.

\section{- Juegos para la estimulación}

Los maestros débén planear los juegos para la éstimulación de acuerdo con sus características sensoriales y motoras. Así se facilitará el cumplimiento de los objetivos de la clase. Por ejemplo, si el objetivo del maestro es lograr agilidad en la carrera, el juego puede ser "perseguidos" o "policías y ladrones". A continuación se darán ejemplos de los juegos que pueden emplear con sus características, objetivos y progresión de acuerdo con la edad de los niños.

\section{- Juegos táctiles}

Los juegos táctiles permiten al niño desarrollar su mapa corporal, gracias a los estímulos que percibe a través de la piel. Son ideales las actividades con arena, agua, pintura, masa, crema, los caminos que ofrecen diversas texturas en las plantas de los pies, los túneles y las cobijas. El énfasis que el maestro puede hacer en estos juegos está en tocar las partes del cuerpo y hablar de su función. Explorar diferentes texturas a través del tacto. Percibir sensaciones de temperatura y textura. E identificar las características de los objetos a través de la manipulación. En el cuadro No. 2 se muestra la progresión por edad, de los juegos tactiles. 


\begin{tabular}{|c|c|}
\hline EDAD & ACTIVIDADES \\
\hline I a 2 años & $\begin{array}{l}\text { Canciones en que el niño se toque cara, manos, pies. Pintarse diferentes partes. del } \\
\text { cuerpo con cremas y pinturas. } \\
\text { Jugar con agua, arena, cremas y diferentes texturas. Piscina de pélotas, mimos. } \\
\text { Dactilopintura. }\end{array}$ \\
\hline 2 a 3 años & $\begin{array}{l}\text { Rondas y canciones que mencionen y se toquen las partes del cuerpo: cabeza, hombros, } \\
\text { rodillas, pies y partes de la cara. } \\
\text { Juegos con agua, arena, dactilopintura y otras texturas: masa, pegantes, barro, plastilina, } \\
\text { etc. } \\
\text { Jugar a los mimos y pintarse la cara. } \\
\text { Jugar a los sandwiches: envolverse en cobijas de diferentes texturas. }\end{array}$ \\
\hline 3 a 5 años & $\begin{array}{l}\text { Juegos con agua, arena, dactilopintura y otras texturas: masa, pegantes, barro, plastilina, } \\
\text { etc. } \\
\text { Jugar en piscina de pelotas. } \\
\text { Jugar a los mimos y pintarse la cara. } \\
\text { Arrastrarse, gatear, caminar descalzos sobre diferentes texturas. } \\
\text { Jugar a los sandwiches: envolverse en cobijas de diferentes texturas. }\end{array}$ \\
\hline 5 años en adelant & $\begin{array}{l}\text { Juegos con agua, arena, dactilopintura y otras texturas: masa, pegantes, barro, plastilina, } \\
\text { etc. } \\
\text { Jugar en piscina de pelotas. } \\
\text { Jugar a los mimos, pintarse la cara. } \\
\text { Arrastrarse, gatear, caminar descalzos en diferentes texturas. } \\
\text { Jugar a los sandwiches: envolverse en cobijas de diferentes texturas. } \\
\text { Descubrir a través del tacto las características dé un objeto: largo, frío, áspero, etc. } \\
\text { Untarse crema, talcos, aceite, vaselina y describir la sensación. } \\
\text { pasar por túneles de diferentes texturas. } \\
\text { Explorar el ambiente por medio del tacto: tocar las cosas y describir cómo son. } \\
\text { Jugar al mago que adivina qué objeto, tiene en la mano. } \\
\text { Describir el objeto que se tiene en las manos, dándole el nombre correcto: es grueso, es } \\
\text { caliente, es áspero. } \\
\text { Jugar en parejas loterías de texturạs. } \\
\text { Pensar qué objetos conocidos en la vida diaria tienen las mismas características del } \\
\text { objeto que sé está analizando. }\end{array}$ \\
\hline
\end{tabular}

Cuadro No. 2 Progresión de los juegos táctiles.

\section{- Juegos de altura y movimiento}

Los juegos de altura y movimiento favorecen las reacciones de equilibrio y desarrollan en el niño la fuerza para mantener posiciones. Se proponen 
actividades en parques, juegos mecánicos, triciclos, rodaderos, balancines, barras de equilibrio, columpios y trampolines. El énfasis que el maestro puede hacer en estos juegos está en experimentar movimientos en diferentes posiciones y direcciones. Favorecer el enderezamiento y el equilibrio. $Y$ promover la buena postura. En el cuadro No. 3 se muestra la progresión por edad de los juegos de altura y movimiento.

\begin{tabular}{|c|c|}
\hline EDAD & ACTIVIDADES \\
\hline 1 a 2 años & $\begin{array}{l}\text { Montar en balancines, Columpios, Rodaderos pequeños } \\
\text { Jugar en rodillos, balones medianos, cojines y colchonetas. } \\
\text { Subir y bajar del gimnasio en diferentes formas: pegados a la escalera, gateando, } \\
\text { rodándose. } \\
\text { Resortar en una tabla estando en posiciones: acostados y sentados } \\
\text { lniciar vueltas en un barril. }\end{array}$ \\
\hline 3 a 5 años & $\begin{array}{l}\text { Montar en balancines, columpios, llantas que cuelgan: aumentar altura de acuerdo } \\
\text { con la edad. } \\
\text { Caminar sobre caminos de cojines, llantas y barra ancha para equilibrio de mayor } \\
\text { altura. } \\
\text { Jugar en balancines y rodadero en diferentes posiciones. } \\
\text { Mecerse en un columpio en diferentes direcciones: iniciar vueltas. }\end{array}$ \\
\hline 5 años en adelante & $\begin{array}{l}\text { Montar en balancines, columpios, llantas que cuelgan: aumentar altura de acuerdo con } \\
\text { la edad. } \\
\text { Caminar sobre caminos de cojines, llantas y barra ancha para equilibrio de mayor } \\
\text { altura. } \\
\text { Jugar en balancines y rodadero en diferentes posiciones. } \\
\text { Mecerse en un columpio en diferentes direcciones: iniciar vueltas. } \\
\text { Caminar por entre líneas pintadas en el suelo, rectas, quebradas y curvas. } \\
\text { Columpiarse solo, girar solo. Dar vueltas y botes. } \\
\text { Jugar en el pasto o sobre terrenos quebrados. }\end{array}$ \\
\hline
\end{tabular}

Cuadro No, 3 Progresión por edad de los juegos de altura y movimiento.

\section{- Juegos de fuerza y plan motor.}

Los juegos de fuerza y plan motor desarrollan los patrones básicos y las habilidades de movimiento. En ellos el niño tiene que plantear estrategias para jugar. Se recomiendan actividades que incluyan secuencias de botes, 
saltos gateo, arrastre, juegos en el gimnasio, el parque, imitar animales con el cuerpo, canciones con mímíca, juegos con muñecos, bailes y dramatizaciones. El énfasis que el maestro puede hacer en estos juegos está en promover el desarrollo adecuado de patrones de locomoción y pretensión. Desarrollar la coordinación. Imprimir velocidad y agilidad a los movimientos. $Y$ resolver problemas de tipo motor. En el cuadro No. 4 se muestra la progresión por edad de los juegós de fuerza y plan motor.

\begin{tabular}{|c|c|}
\hline EDAD & ACTIVIDADES \\
\hline I a 2 años & $\begin{array}{l}\text { Oír música y moverse al ritmo de ella. Rondas cortas } \\
\text { Juegos como estatuas y Simón dice. Juegos en el espejo. Mímica. } \\
\text { Empujar coches, carros, cajas vacías y llenas. } \\
\text { Cargar objetos pesados y livianos: bolsas de arena, cajas, etc. } \\
\text { Alcanzar objetos. Meter y sacar objetos de una caja. } \\
\text { Ensamblar y arrojar objetos. } \\
\text { lnstrucciones cortas. Ej. : manos a la cabeza, tírate por el rodadero, etc. } \\
\text { Gatear sobre diferentes superficies: colchonetas gruesas y delgadas, cojines, } \\
\text { espumas, etc. }\end{array}$ \\
\hline 2 a 3 años & $\begin{array}{l}\text { Rondas con movimientos al ritmo de la música. } \\
\text { Juegos en los que se incluya cambio de posición : acostarse, sentarse, pararse. } \\
\text { Juegos en que se incluyen desplazamientos: caminar adelante, atrás, al lado. } \\
\text { Juegos con pesos: bolsas de arena, cajas, etc. } \\
\text { Juegos de empujar: llantas, coches, cojines, cajas. } \\
\text { Pasar por caminos con obstáculos. } \\
\text { Juego de imitar el caminado de los animales : arrastre, gateo, caminar, correr. } \\
\text { Juego de estatuas. Juegos de Simón dice. } \\
\text { Pasar por pequeños obstáculos : cojines, llantos, aros. } \\
\text { Jugar con balones y aros } \\
\text { Juegos de seguir instrucciones cortas. }\end{array}$ \\
\hline 3 a 5 años & $\begin{array}{l}\text { Moverse al ritmo de la música. Rondas y juegos en que el cuerpo cambie de posición. } \\
\text { Juegos con pesos : lanzar y recoger. } \\
\text { Pasar por el gimnasio : agacharse, pararse, trepar y saltar. Colgarse. } \\
\text { Halar lazos. Empujar pesos. Llevar pesos sobre el cuerpo. } \\
\text { Arrastrarse sobre carros de balineras } \\
\text { Juego de estatuas y Simón dice. Imitar posiciones. } \\
\text { Juegos con instrumentos: correr al sonido, parar en el silencio. } \\
\text { Cuadro No. } 4 \text { Continua... }\end{array}$ \\
\hline
\end{tabular}




\begin{tabular}{|c|c|}
\hline EDAD & ACTIVIDADES \\
\hline 3 a 5 años & $\begin{array}{l}\text { Lanzar a un blanco: usando manos y pies. } \\
\text { Realizar secuencias motoras imitando al primero. } \\
\text { Realizar instrucciones motoras: súbete a los cojines, pasa por la escalera, etc. }\end{array}$ \\
\hline 5 años en adelante & $\begin{array}{l}\text { Moverse al ritmo de la música. Jugar al moverse al tiempo con una canción que } \\
\text { describa movimiento. } \\
\text { Juegos con instrumentos: correr al sonido, parar en el silencio. } \\
\text { Hacer secuencias motoras al estilo de un animal. } \\
\text { Jugar a manejar ritmos lentos y rápidos y hacer que los niños los analicen. Explorar } \\
\text { diferentes pesos y tamaños. Empujar cosas y llevar pesos sobre el cuerpo. } \\
\text { Juegos con balones : lanzar y recoger } \\
\text { Jugar en parejas: alzarse, empujarse, llevarse en carretilla } \\
\text { Jugar a medir la fuerza que hacen : poca, mucha. } \\
\text { Trepar y escalar. Colgarse en barras. Halar lazos. } \\
\text { Arrastrarse sobre carro de balineras. } \\
\text { Imprimir velocidad en los juegos motores y secuencias. } \\
\text { Hacer carreras cambiando de dirección cuando se indique. } \\
\text { Juego de estatuas y Simón dice. Jugar cruz roja. Imitar posiciones y movimientos. } \\
\text { Carros con semáforos en rojo y verde } \\
\text { Caminar por un camino delineado en el piso. } \\
\text { Lanzar a un blanco usando manos y pies. } \\
\text { Realizar secuencias motoras imitando al primero } \\
\text { Hace dramatizaciones de oficios. Juegos de expresión corporal en que se va diciendo } \\
\text { qué hacer, etc. } \\
\text { Recordar dos o tres instrucciones y realizarlas. }\end{array}$ \\
\hline
\end{tabular}

Cuadro No. 4 Progresión por edad de los juegos de fuerza y plan motor.

\section{- Juegos para coordinar los dos lados del cuerpo}

Los juegos para coordinar los dos lados del cuerpo favorecen el proceso de bilateralidad. El uso armónico de las manos y la preferencia manual. Son buenas las actividades con balones, bates, raquetas, carritos, bolos, tiro al blanco, construcciones con fichas, cargar objetos grandes y encajar o guardar cosas. El énfasis que el maestro puede hacer en estos juegos está en favorecer el desarrollo de destrezas bilaterales. Promover el cruce de la línea media y 


\section{el uso dé la mano preferente. En el cuadro No. 5 se muestra la progresión por edad de los juegos para manejar los dos lados del cuerpo.}

\begin{tabular}{|c|c|}
\hline EDAD & ACTIVIDADES \\
\hline I a 2 años & $\begin{array}{l}\text { Pasar por colchoneta, túneles y obstáculos pequeños: cojines, almohadas, rollitos. } \\
\text { Subir y bajar escaleras pequeñas. } \\
\text { Llenar cajas con objetos. } \\
\text { Juegos con bloques grandes: cargar, soltar. } \\
\text { Juegos con pelotas grandes y pequeñas: lanzar, patear. }\end{array}$ \\
\hline 2 a 3 años & $\begin{array}{l}\text { Caminos imitando el caminado de animales: serpiente, oso, gato, conejo, caballo, etc. } \\
\text { Caminos con obstáculos: túnel, cojines, llantas, rodadero. } \\
\text { Jugar en el gimnasio pequeño. } \\
\text { Ensamblar fichas grandes y medianas. } \\
\text { Jugar con pelotas grandes y pequeñas. }\end{array}$ \\
\hline 3 a 5 años & $\begin{array}{l}\text { Juegos de obstáculos y caminos usando túneles, llantas, cojines, etc. en que se puedan } \\
\text { usar los diferentes patrones. } \\
\text { Jugar en el gimnasio. } \\
\text { Saltar obstáculos. } \\
\text { Encestar, recibir, patear pelota. } \\
\text { Construir con fichas medianas. } \\
\text { Lanzar tiro al blanco. Jugar bolos. }\end{array}$ \\
\hline 5 años en adelante & $\begin{array}{l}\text { Aprender diferentes juegos tradicionales: golosa, rondas, lazo. } \\
\text { Juegos de obstáculos y caminos usando túneles, llantas, cojines, etc. en que se puedan } \\
\text { usar los diferentes patrones. } \\
\text { Jugar a imitar los movimientos de los animales. } \\
\text { Jugar en el gimnasio. } \\
\text { Hacer dramatizaciones de los oficios. } \\
\text { Saltar obstáculos. } \\
\text { Jugar en el parque en diferentes superficies. } \\
\text { Encestar, recibir, patear pelota. } \\
\text { Lanzar tiro al blanco. Jugar bolos. } \\
\text { Armar con fichas grandes. }\end{array}$ \\
\hline
\end{tabular}

Cuadro No. 5

Progresión por edad, de los juegos para coordinar los dos lados del cuerpo. 


\section{- Juegos para manejar el espacio}

Los juegos para manejar el espacio promueven en el niño la organización del espacio que lo rodea. Se proponen actividades como seguir al primero, encontrar pistas, ordenar objetos, jugar por caminos de acuerdo con una instrucción y juegos de expresión corporal. Durante los juegos, los adultos deben hacer señalamiento sobre la posición de los objetos o la dirección que se debe tomar en una actividad. El énfasis que el maestro puede hacer en estos juegos está en organizar conceptos espaciales a través del movimiento. Desarrollar la direccionalidad. Promover la creación de relaciones espaciales. $Y$ favorecer la coordinación ojo-mano y ojo-pié. En el cuadro No. 8 se muestra la progresión por edad de los juegos para manejar el espacio.

\begin{tabular}{|c|c|}
\hline EDAD & ACTIVIDADES \\
\hline 1 a 2 años & $\begin{array}{l}\text { Juegos con cambios de dirección y posición. } \\
\text { Empujar cojines, halar cosas y llevadas a un lugar. } \\
\text { Pasar por caminos con obstáculos: túneles, montañas de cojines, rodaderos, llantas, } \\
\text { etc. Mientras se les dice la dirección que toman. } \\
\text { Empezara hablar de posiciones: arriba, abajo, adelante y atrás. }\end{array}$ \\
\hline 2 a 3 años & $\begin{array}{l}\text { Pasar por caminos de obstáculos. } \\
\text { Jugar con aros. } \\
\text { Jugar en el gimnasio, hablándoles de la posición. } \\
\text { Inventar formas de salir de un sitio, rodarse, trepar, saltar, etc. } \\
\text { Caminair por una secuencia mientras se les habla de la dirección que toman. Empezar } \\
\text { a hablar de: arriba, abajo, adelante y atrás, encima, debajo, afuera adentro. }\end{array}$ \\
\hline 3 a 5 años & $\begin{array}{l}\text { Pasar por caminos de obstáculos: } \\
\text { Jugar con aros. } \\
\text { Jugar en el gimnasio, hablándoles de la posición. } \\
\text { Inventar formas de salir de un sitio, rodarse; trepar, saltar, etc. } \\
\text { Caminar por una secuencia mientras se les habla de la dirección que toman. } \\
\text { Ordenar objetos por color, forma, tamaño. } \\
\text { Llévar y traer objetos de un lugar a otro. } \\
\text { Enfatizar posiciones de: arriba, abajo, adelante y atrás, encima, debajo, afuera, } \\
\text { adentro, a través y alrededor. }\end{array}$ \\
\hline & Cuadro No 6 Continua... \\
\hline
\end{tabular}




\begin{tabular}{|l||l||}
\hline \multicolumn{1}{|c||}{ EDAD } & \multicolumn{1}{|c|}{ ACTIVIDADES } \\
\hline \hline años en adelante & $\begin{array}{l}\text { Pasar por caminos de obstáculos. } \\
\text { Jugar con aros. } \\
\text { Jugar en el gimnasio, hạblándoles de la posición. } \\
\text { Inventar formas de salir de un sitio, rodarse, trepar, saltar, etc. } \\
\text { Caminar por una secuencia mientras se les habla de la dirección que toman. } \\
\text { Ordenar objetos por color, forma, tamaño. } \\
\text { Llevar y traer objetos de un lugar a otro. } \\
\text { Pasar por parejas a cumplir una instrucción relacionada con posición o dirección. } \\
\text { Recordar el orden en que se hizo un juego. } \\
\text { Enfatizar posiciones de : arriba, abajo, adelante y atrás, encima, debajo, afuera, adentro, } \\
\text { a través, alrededor, derecha e izquierda. }\end{array}$ \\
\hline
\end{tabular}

Cuadro No. 6 Progresión por edad de los juegos para manejar el espacio. 


\section{CONCLUSIONES}

El juego es una estrategia de enseñanza de gran utilidad para favorecer el desarrollo motor del niño. Durante el juego el niño logra el control de los movimientos de su cuerpo y adquiere coordinación y destreza. La interacción con los juguetes ofrece una gran variedad de estímulos sensoriales que promueven por ejemplo, la adquisición del mapa corporal, la base postural y la organización en el espacio. En la escuela, los maestros pueden utilizar el recurso del juego como facilitador del aprendizaje infantil, ya que es fuente de motivación y permite el afianzamiento de las habilidades motoras. Una adecuada preparación del ambiente escolar en términos lúdicos permitirá simultáneamente el cumplimiento de objetivos pedagógicos y el desarrollo de habilidades sensoriomotoras. Las actividades escolares que tienen que ver con el desarrollo de habilidades motoras pueden ser más atractivas para los niños si se plantean en términos de juego, facilitando al maestro el cumplimiento de su labor escolar. 


\section{BIBLIOGRAFÍA}

1. Amaya, Mariluces. Lizarazo, R., Restrepo, B. Tobón, M. Trujillo, C. (1996). Modelo de pedagogía participativa para programas de educación preescolar. Asociación Colombiana de Terapia Ocupacional, (Editores), Revista Ocupación Humana. Volúmen 6. Número 4 de 1996. Santafé de Bogotá.

2. Bundy, Anita (1991). Play Theory and Sensory lntegration. A. Fisher, Murray y A. Bundy (Editores). Sensory Integration, Theory and Practice, F.A. Davis Company. Philadelphia.

3. Bundy, Anita (1994). Test of Playfullness Research version. Colorado State University. FT. Collins Colorado.

4. Durán Acosta, José A. (1994), El Proyecto educativo Institucional, una alternativa para el desarrollo pedagógico cultural. Ed. Magisterio: Santafé de Bogotá.

5. Fisher, Anne (1991) Editora. Sensory Integration Theory and Practice. F.A. Davis Company. Philadelphia.

6. Gassier, Jacqueline (1983). Manual de Desarrollo psicomotor del niño. Toray - Masson, S.A. Barcelona.

7. Gjuth, Hans. (1978). La Teoría de Piagiet en la práctica. Una escuela para pensar. Editorial Kapeluz. Buenos Aires.

8. Kielhofner, Gary. (1992). Conceptual Fundations of Occupational Therapy. F.A. Davis Company. Philadelphia.

9. Mc Ewen, Molly (1990). The Human Environment Interface in Occupational Therapy. S. Cook (Editora). Enviroment Implications for 
Occupational Therapy Practice, The American Occupational Therapy Association, lnc. Rockville, Maryland.

10. Richter, Eileen (1 990). Environmental Matrices for Sensory Integrative Treatment. S. Cook (Editora) Enviroment Implications for Occupational Therapy Practice. The American Occupational Therapy Association, Inc. Rockville, Maryland.

11. Pérez, Gloria (1 993). Elaboración de Proyectos Sociales, Casos Prácticos. Narcea, S.A. Ediciones. Madrid.

12. Reilly, Mary (1974). Editora. Play as a Exploratory Learning. Beverly Hills. Sage. 


\section{SUMARY}

Play is a child's most serios occupation. This is a way a child uses any situation to play. Through play a child learns about the world around him. Through play a child acquires abilities to interact with his environment.

During the first school years child's play is focused in movement activities (motor skill development). Through play a child develops and exercises his gross motor skills and improves coordination and ability. He also recives many sensory stimulee from his surroundings. These stimulee will help the child develope body awareness, coordination, spatial organization, strenght and body posture. In other words, play promotes life skills.

Due to these important reasons is very useful to give ideas for fun and games to the teachers. These teaching alternatives which will provide learning through play. 